\title{
MAP4K3/GLK in autoimmune disease, cancer and aging
}

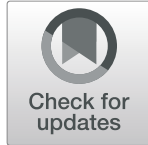

Huai-Chia Chuang ${ }^{1}$ and Tse-Hua $\operatorname{Tan}^{1,2^{*}}$ (D)

\begin{abstract}
MAP4K3 (also named GLK) is a serine/threonine kinase, which belongs to the mammalian Ste20-like kinase family. At 22 years of age, GLK was initially cloned and identified as an upstream activator of the MAPK JNK under an environmental stress and proinflammatory cytokines. The data derived from GLK-overexpressing or shRNAknockdown cell lines suggest that GLK may be involved in cell proliferation through mTOR signaling. GLK phosphorylates the transcription factor TFEB and retains TFEB in the cytoplasm, leading to inhibition of cell autophagy. After generating and characterizing GLK-deficient mice, the important in vivo roles of GLK in T-cell activation were revealed. In T cells, GLK directly interacts with and activates PKC $\theta$ through phosphorylating PKC $\theta$ at Ser-538 residue, leading to activation of IKK/NF-KB. Thus, GLK-deficient mice display impaired T-cell-mediated immune responses and decreased inflammatory phenotypes in autoimmune disease models. Consistently, the percentage of GLK-overexpressing $T$ cells is increased in the peripheral blood from autoimmune disease patients; the GLK-overexpressing T cell population is correlated with disease severity of patients. The pathogenic mechanism of autoimmune disease by GLK overexpression was unraveled by characterizing T-cell-specific GLK transgenic mice and using biochemical analyses. GLK overexpression selectively promotes IL-17A transcription by inducing the AhRRORyt complex in T cells. In addition, GLK overexpression in cancer tissues is correlated with cancer recurrence of human lung cancer and liver cancer; the predictive power of GLK overexpression for cancer recurrence is higher than that of pathologic stage. GLK directly phosphorylates and activates IQGAP1, resulting in induction of Cdc42mediated cell migration and cancer metastasis. Furthermore, treatment of GLK inhibitor reduces disease severity of mouse autoimmune disease models and decreases IL-17A production of human autoimmune T cells. Due to the inhibitory function of HPK1/MAP4K1 in T-cell activation and the promoting effects of GLK on tumorigenesis, HPK1 and GLK dual inhibitors could be useful therapeutic drugs for cancer immunotherapy. In addition, GLK deficiency results in extension of lifespan in Caenorhabditis elegans and mice. Taken together, targeting MAP4K3 (GLK) may be useful for treating/preventing autoimmune disease, cancer metastasis/recurrence, and aging.
\end{abstract}

Keywords: MAP4K3 (GLK), HPK1, Autoimmune disease, Cancer metastasis, Aging, IL-17A, PKCO, IQGAP1, Autophagy, Verteporfin

\section{Background}

The MAP4K (MAP kinase kinase kinase kinase or MAPKKKK) family kinases are serine/threonine kinases, which belong to the mammalian Ste20-like kinase family $[1,2]$. Overexpression of MAP4K family kinases specifically induces activation of JNK [3-5], but not p38 or ERK [6], in mammalian cells [1]. The MAP4K family contains seven members, including MAP4K1/HPK1 (hematopoietic

\footnotetext{
* Correspondence: ttan@nhri.edu.tw

${ }^{1}$ Immunology Research Center, National Health Research Institutes, 35 Keyan Road, Zhunan 35053, Taiwan

${ }^{2}$ Department of Pathology \& Immunology, Baylor College of Medicine, Houston, TX 77030, USA
}

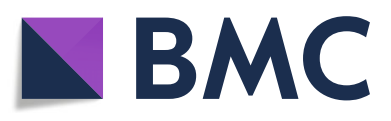

(c) The Author(s). 2019 Open Access This article is distributed under the terms of the Creative Commons Attribution 4.0 International License (http://creativecommons.org/licenses/by/4.0/), which permits unrestricted use, distribution, and

reproduction in any medium, provided you give appropriate credit to the original author(s) and the source, provide a link to the Creative Commons license, and indicate if changes were made. The Creative Commons Public Domain Dedication waiver (http://creativecommons.org/publicdomain/zero/1.0/) applies to the data made available in this article, unless otherwise stated. ter kinase)/RAB8IP [9], MAP4K3/GLK (GCK-like kinase) [10], MAP4K4/HGK (HPK1/GCK-like kinase) [11, 12], MAP4K5/KHS (kinase homologous to SPS1/Ste20) [13], MAP4K6/MINK (misshapen/Nck-related kinase) [14], and MAP4K7/TNIK (TRAF2 and NCK interacting kinase) [15]. In 1997, MAP4K3 (GLK) was cloned and identified as a protein kinase that shares $49 \%$ amino acid identity with MAP4K1/HPK1 kinase domain and 57\% amino acid identity with MAP4K2/GCK kinase domain, thus named GCK-like kinase (GLK) [10]. Like MAP4K1 (HPK1) protein, GLK protein contains a conserved amino-terminal 
kinase domain, three proline-rich motifs, and a conserved carboxy-terminal citron-homology domain (Fig. 1) [10]. MAP4K1 (HPK1) contains a caspase-3 recognition site (Asp-Asp-Val-Asp, amino acids 382-385) [16]. MAP4K3 (GLK) also contains a consensus sequence (Asp-Glu-GlyAsp, amino acids 415-418) that matches the substrate sequence Asp-Glu-X-Asp for caspase 2, 3, or 7 [17], suggesting that GLK protein may also be cleaved by a caspase. Overexpression of GLK induces its autophosphorylation and its kinase activity [10]; Ser-170 residue is identified as the trans-autophosphorylation site of GLK protein [18] (Fig. 2). MAP4K3 (GLK) was initially identified as an upstream activator for JNK activation under an environmental stress and proinflammatory cytokines [10]. MAP4K3 (GLK) overexpression induces JNK activation in HEK293T human embryonic kidney cells through MEKK1 [10]. Further studies using knockout/transgenic mice or biochemical approaches reveal additional GLK functions, which are described in this review.

\section{MAP4K3 (GLK) induces mTOR signaling and inhibits autophagy}

MAP4K3 (GLK) controls the cilium growth/development of Caenorhabditis elegans and the wing development of Drosophila melanogaster through mTOR signaling $[19,20]$. Overexpression of MAP4K3 (GLK) induces activation of the mTOR downstream molecules S6K and 4E-BP1 in Hela cells upon sensing cellular nutrient and energy levels; conversely, GLK siRNA knockdown inhibits the activation of S6K and 4E-BP1 [21].
Moreover, like treatment of the mTOR inhibitor rapamycin, GLK siRNA knockdown also inhibits cell growth of Hela cells [21]. In addition, MAP4K3 (GLK) directly interacts with and phosphorylates the transcription factor TFEB at Ser-3 residue, resulting in inhibition of amino acid-depletion-mediated TFEB nuclear translocation [22]. The GLK-induced TFEB Ser-3 phosphorylation is required for the subsequent Ser-211 phosphorylation of TFEB by mTORC1, leading to retention of TFEB in the cytosol and inhibition of cell autophagy [22]. Notably, the GLK-mediated TFEB inactivation facilitates the mTORinhibited autophagy pathway, but the TFEB inactivation is not regulated by mTOR signaling [22]. Besides induction of mTOR signaling, GLK overexpression induces NF- $\mathrm{kB}$ activation [23] and cell proliferation in primary human hepatocytes [24]. These findings suggest that MAP4K3 (GLK) plays critical roles in promoting cell growth and blocking autophagy (Fig. 3).

\section{Upstream regulators of MAP4K3 (GLK)}

Upon amino acid withdrawal, the phosphatase PP2A directly interacts with and dephosphorylates GLK at the autophosphorylation site Ser-170, leading to inactivation of GLK and GLK-induced mTOR signaling [18]. Besides serine phosphorylation, tyrosine phosphorylation of GLK is induced by EGF stimulation in A549 lung cancer cell lines, suggesting that EGF receptor (EGFR) signaling regulates GLK function or activity [25]. Moreover, Tyr-366, Tyr-379, Tyr-574, and Tyr-735 are identified as the induced tyrosine-phosphorylation residues of GLK in cells treated
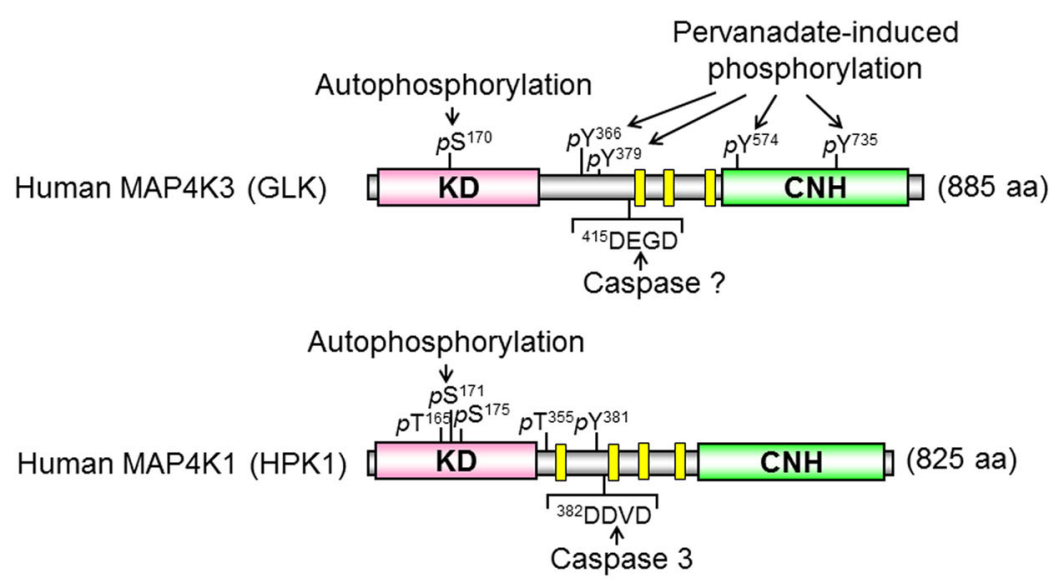

KD Kinase domain Proline-rich motif CNH Citron-homology domain

Fig. 1 The structural domains of human MAP4K3 (GLK) and human MAP4K1 (HPK1). MAP4K family kinases such as MAP4K3 (GLK) and MAP4K1 (HPK1) are composed of a kinase domain (KD), proline-rich motifs in the middle region, and a citron-homology (CNH) domain. The autophosphorylation sites of GLK and HPK1 are phospho-Ser-170 residue and phospho-Ser-171 residue, respectively. Four pervanadate-induced tyrosine phosphorylation residues on GLK proteins and the known phosphorylation residues on HPK1 proteins are also indicated. The caspase-3 cleavage site on HPK1 and a putative caspase cleavage site on GLK are indicated 


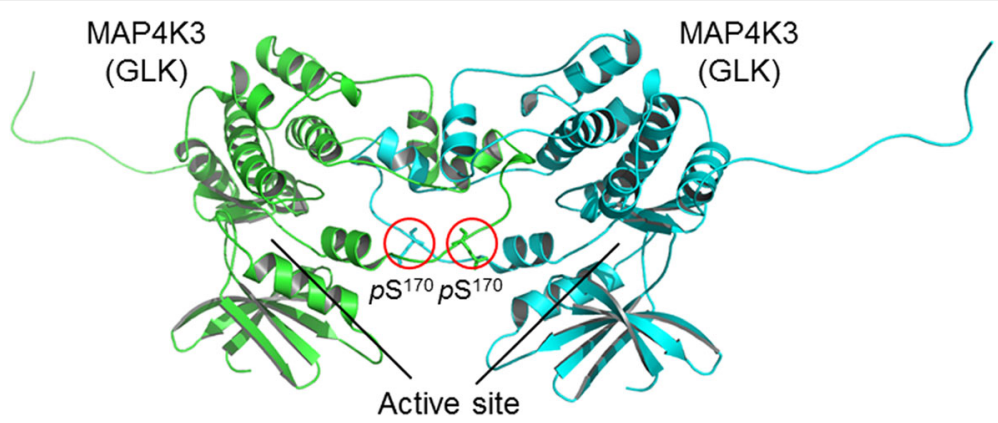

Fig. 2 A three-dimensional structure model depicts the dimerization of two MAP4K3 (GLK) kinase domains containing the phospho-Ser-170 residues. Circles denote the phosphates on the Ser-170 residues. The active site of MAP4K3 (GLK) kinase domain is indicated

with the tyrosine phosphatase inhibitor pervanadate [26]. In addition, the adaptor protein HIP-55 (also named mAbp1 and SH3P7) has been identified as an interacting protein of MAP4K3 (GLK) and HPK1 (MAP4K1) [27]. Both GLK and HIP-55 are required for T-cell activation [23, 28]. Furthermore, HIP-55 overexpression stimulates HPK1 kinase activity [27]; conversely, T-cell receptor (TCR)-induced HPK1 activation is reduced in HIP-55 knockout T cells [28]. The data suggest that GLK may also be a downstream molecule of HIP-55. Like HPK1 [29], GLK directly interacts with the adaptor protein SLP-76 under TCR signaling [23]. SLP-76 is required for TCR-induced GLK kinase activity [23]; however, the kinase that phosphorylates and regulates GLK has not been identified.

\section{MAP4K3 (GLK) controls T-cell activation and T- cell-mediated autoimmune responses}

After generating and characterizing GLK-deficient mice, the in vivo roles of GLK in T-cell activation have been revealed. GLK-deficient mice display impaired T-cellmediated immune responses [23]. In T cells, GLK kinase activity is induced by T-cell receptor (TCR) stimulation [23]. Under TCR signaling, GLK directly interacts with and activates PKC $\theta$ through phosphorylating PKC $\theta$ at Thr-538 residue but not Ser-676 and Ser-695 residues $[23,30]$, leading to activation of IKK/NF- $\mathrm{kB}$ [23]. In vitro Th1, Th2, or Th17 differentiation is reduced by GLK deficiency [23]. In contrast, suppressive function of GLK-deficient Treg cell is enhanced compared to that of wild-type Treg cell [23]. Thus, GLK positively regulates $\mathrm{T}$-cell activation and T-cell function by activating the PKCO-IKK pathway (Fig. 3).

GLK overexpression contributes to autoimmune responses. GLK-deficient mice display decreased disease scores in autoimmune disease models such as collageninduced arthritis (CIA) [31] and experimental autoimmune encephalomyelitis (EAE) [23]. Consistently, the percentage of GLK-overexpressing T cells is enhanced in the peripheral

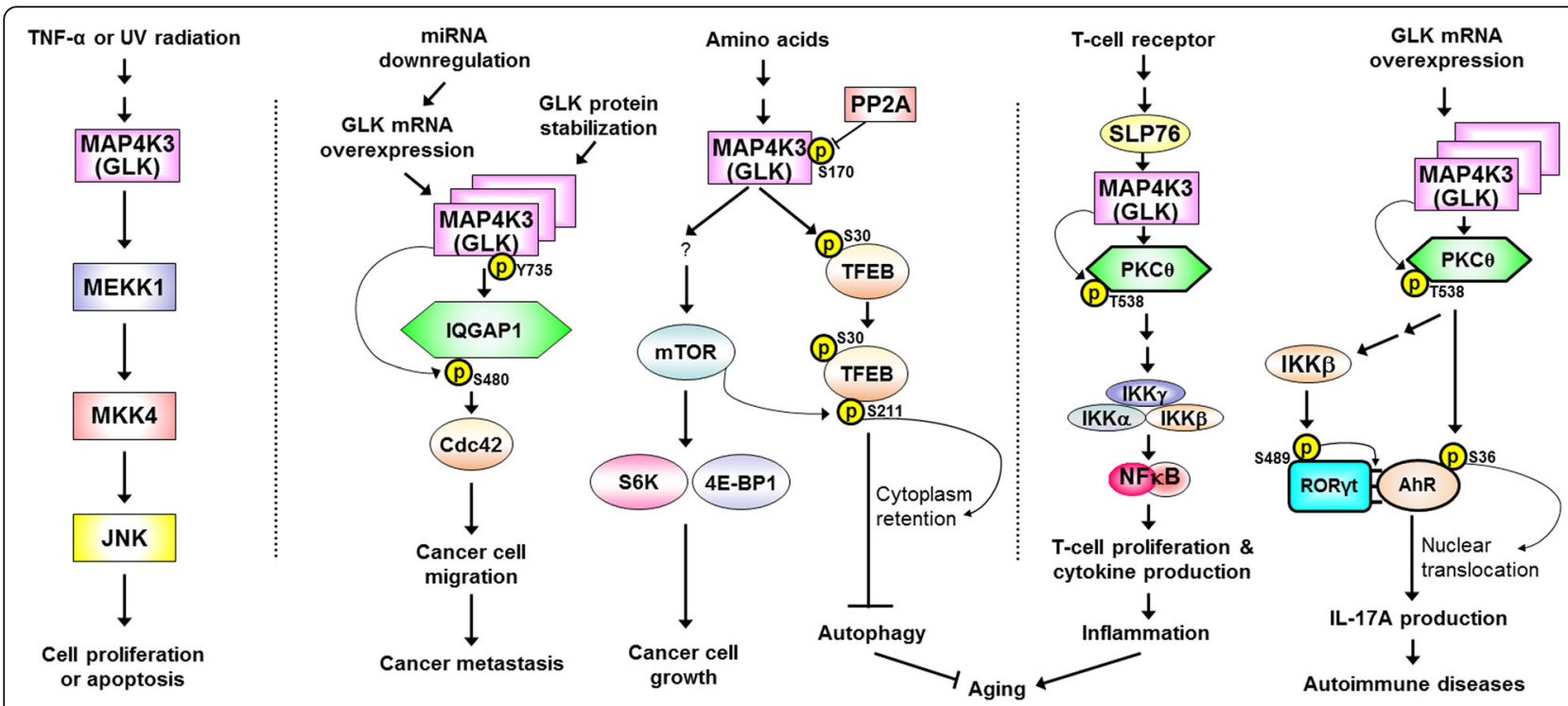

Fig. 3 Summary of signal transduction pathways of MAP4K3 (GLK). Arrows denote activation; T bar denotes inhibition. GLK signaling pathways in TNF-a signaling and apoptosis (left panel), cancer and autophagy (middle panel), as well as TCR signaling and autoimmunity (right panel) 
blood from patients with human autoimmune diseases, including systemic lupus erythematosus (SLE) [23], rheumatoid arthritis (RA) [31], and adult-onset Still's disease (AOSD) [32]. The GLK-overexpressing T cell population is correlated with disease severity of autoimmune disease patients [23, 31, 32]; therefore, GLK-overexpressing $\mathrm{T}$ cell is a biomarker for autoimmune diseases.

To mimic GLK overexpression in human autoimmune patient $\mathrm{T}$ cells, $\mathrm{T}$-cell-specific GLK transgenic mice have been generated and characterized. The transgenic mice spontaneously develop autoimmune diseases and systemic inflammation [33]. The pathogenic cytokine IL-17A levels are specifically increased in the sera of T-cell-specific GLK transgenic mice [33]. Consistently, GLK overexpression co-exists with IL-17A overproduction in peripheral blood $\mathrm{T}$ cells from human SLE patients [34]; the $\mathrm{GLK}{ }^{+} \mathrm{IL}-17 \mathrm{~A}^{+}$ $T$ cell population is a diagnostic biomarker for active SLE [34]. The pathogenic mechanism of autoimmune disease by GLK overexpression has been unraveled using several gene knockout/transgenic mice and biochemical approaches [33]. In T cells, GLK overexpression induces activation of PKC $\theta$ and IKK $\beta$ [33]. IKK $\beta$ phosphorylates ROR $\gamma t$ at Ser-489 residue, leading to the interaction between the transcription factors RORyt and AhR [33]. On the other hand, PKC $\theta$ phosphorylates AhR at Ser-36 residue, resulting in nuclear translocation of the AhR-ROR $\gamma \mathrm{t}$ complex [33]. Thus, GLK overexpression in T cells selectively promotes IL-17A transcription by inducing the AhRRORyt complex (Fig. 3). The GLK-regulated AhR-RORyt complex is also induced in peripheral blood $\mathrm{T}$ cells from human SLE patients [34]. Conversely, AhR or ROR $\gamma t$ deficiency results in attenuation of autoimmune responses of T-cell-specific GLK transgenic mice [33]. These findings indicate that GLK signaling and GLK-induced AhRRORyt complex are potential therapeutic targets for SLE.

\section{MAP4K3 (GLK) promotes cancer metastasis/ recurrence}

GLK overexpression occurs in cancer tissues of human non-small cell lung carcinoma (NSCLC) [35], hepatocellular carcinoma (HCC) [24], glioblastoma [36], and papillary thyroid carcinoma (PTC) [37]. One publication reported that GLK immunohistochemistry (IHC) staining signals per square microns are decreased in cancer tissues of pancreatic cancer patients [38]. Nevertheless, IHC staining intensity of GLK seems to be more condensed in ductal adenocarcinoma-like cells, which would be consistent with others' findings that GLK is overexpressed in several cancer tissues [24, 35-37]. Moreover, a GLK somatic mutation, encoding $\mathrm{E} 351 \mathrm{~K}$, has been identified in human pancreatic cancer [39]. The GLK E351K mutant displays higher kinase activity than that of wild-type GLK [26], indicating that GLK is an oncogene involved in tumorigenesis of human pancreatic cancer.
For human NSCLC and HCC, GLK overexpression in cancer tissues is correlated with cancer recurrence $[24,35]$. The predictive power of GLK overexpression for cancer recurrence is higher than that of pathologic stage. Another MAP4K member, MAP4K4/HGK, induces cell migration and promotes cancer metastasis [40]. It is likely that GLK may also promote cancer metastasis by enhancing cell migration. In addition, overexpression of the microRNAs let7c, miR-199-a-5p, or miR-206 inhibits GLK expression in cell lines by targeting the GLK 3'UTR [37, 41, 42]. Overexpression of let-7c or miR199-a-5p also inhibits cell migration and invasion of cancer cell lines [41, 42]. Consistently, cell migration and invasion are also attenuated by GLK siRNA knockdown but enhanced by GLK overexpression in liver cell lines [42]. Moreover, the two aforementioned miRNAs (let-7c and miR-199-a-5p) are downregulated in tumor tissues of human NSCLC and HCC, respectively, and the downregulation of these two miRNAs is correlated with poor outcome of cancer patients [41, 42]. These results suggest that GLK may induce cancer metastasis/recurrence of NSCLC and HCC by enhancing cell migration/invasion.

A recent publication reveals the mechanism of GLKinduced cell migration and cancer metastasis using wholebody GLK transgenic mice [26] (Fig. 3). GLK transgene induces cell migration in primary lung epithelial cells [26]. GLK transgene does not initiate tumorigenesis in mice [26]; however, GLK transgene promotes distant cancer metastasis in a genetically modified lung cancer mouse modellung-specific EGFR-deletion mutant transgenic mouse line (EGFR ${ }^{\text {del }} \mathrm{Tg}$ ) [26]. The GLK-interacting protein IQGAP1 is responsible for GLK-induced cell migration and cancer metastasis [26]. GLK directly interacts with and phosphorylates IQGAP1 at Ser-480 residue, resulting IQGAP1 activation and subsequent cell migration [26]. Furthermore, Ser-480 phosphorylated IQGAP1 induces activation of Cdc42, which controls non-directional/random cell migration [26]. In contrast, GLK-phosphorylated IQGAP1 does not activate another IQGAP1-downstream molecule, Rac1, which controls directional/persistent cell migration. In addition, the direct interaction between GLK and IQGAP1 is mediated by two proline-rich regions of GLK and the WW domain of IQGAP1; this interaction also is inducible by GLK Tyr-735 phosphorylation [26]. GLK and IQGAP1 colocalize at the leading edge of migrating cells [26]. Consistently, GLK-IQGAP1 interaction and GLK-induced IQGAP1 Ser-480 phosphorylation are detectable in cancer tissues and metastatic cancer cells of human lung cancer patients; this interaction and IQGAP1 phosphorylation are correlated with poor survival of lung cancer patients [26]. Conversely, the distant cancer metastasis is abolished by IQGAP1 knockout in a cancer mouse model [26]. Collectively, the GLK-IQGAP1 complex and IQGAP1 Ser-480 phosphorylation are prognostic biomarkers and potential therapeutic targets for human lung cancer recurrence. 
Besides intrinsic pathways, GLK overexpression may contribute to metastasis through proinflammatory cytokines. GLK overexpression in T cells induces production of IL-17A [33], which promotes cancer cell migration and increases cancer metastasis [43-45]. Therefore, GLK overexpression in $\mathrm{T}$ cells or other cell types may also induce overproduction of IL-17A in tumor microenvironment, leading to cancer metastasis.

\section{GLK inhibitors for treatment of Th17-mediated autoimmune diseases}

GLK overexpression is a therapeutic target for autoimmune diseases and cancer recurrence. Inhibition of GLK may be useful for treating cancer and autoimmune disease patients. An analogue of crizotinib (compound \#1) has been identified as a small-molecule GLK inhibitor that binds to the active site of the GLK kinase domain [46]. The IC50 for GLK kinase activity by the crizotinib analogue is $10 \mathrm{nM}$; however, the IC50 for MAP4K4 (HGK) is $0.8 \mathrm{nM}$ [46]. MAP4K4 (HGK) is a negative regulator for Th17 development [47-49]; therefore, preferential inhibition of MAP4K4 (HGK) by this crizotinib analogue (compound \#1) may result in the adverse effect of inducing Th17-mediated immune responses, such as autoimmunity. These results suggest that this crizotinib analogue may not be useful for treatment of autoimmune disease. Another crizotinib analogue (compound \#44) was also identified as a GLK inhibitor with IC50 of $3 \mathrm{nM}$, but it still inhibits other MAP4K members [50]. Furthermore, in vivo clearance rate in animals of this crizotinib analogue (compound \#44) is too high to test its inhibitory effects using animal models [50]. Thus, the authors concluded that no promising GLK inhibitors have been developed from the analogues of crizotinib [50].

Recently, an FDA-approved drug, verteporfin, has been identified as a new small-molecule GLK inhibitor [34]. Verteporfin is a light-activated drug for macular degeneration of eyes [51]. The IC50 of verteporfin for GLK kinase activity is $1.15 \mathrm{nM}$ without any photochemical process, while the IC50 of verteporfin for HPK1 (MAP4K1) kinase activity is $7.91 \mathrm{nM}$ [34]. Notably, the IC50 of verteporfin for MAP4K3 (GLK) is the lowest compared to that of other MAP4K members [34]. The verteporfin treatment reduces disease severity in three autoimmune mouse models, including EAE, CIA, and T-cell-specific GLK transgenic mice [34]. Moreover, the verteporfin treatment also efficiently inhibits GLK-induced AhR-RORyt complex and IL-17A production in human SLE T cells [34]. Thus, verteporfin may be repositioned as a novel small-molecule therapeutic drug for Th17-mediated autoimmune diseases.

\section{GLK inhibitors for treatment of cancer recurrence} The correlation between GLK overexpression and cancer recurrence and the promotion of cancer metastasis by
GLK suggest that the GLK inhibitor verteporfin is also useful for treating cancer. Furthermore, verteporfin has also been used in clinical trials for pancreatic cancer due to its inhibitory effect on angiogenesis by releasing reactive oxygen radicals [52]. Thus, verteporfin is a potential therapeutic drug for both autoimmune disease and cancer recurrence.

Natural products also contains GLK inhibitors [53]. Astragalus polysaccharide (APS) and 10-hydroxycamptothecin (HCPT) have been reported as GLK inhibitors that suppress GLK kinase activity and GLK-induced mTOR signaling [53]. Combination treatment of APS and HCPT induces cell apoptosis and reduces cell migration/invasion in H1299 lung cancer cells [53]. Further purification of APS and HCPT may help development of small-molecule GLK inhibitors for treatment of cancer recurrence and autoimmune diseases.

\section{GLK and HPK1 dual inhibitors for cancer immunotherapy}

A higher dose $(7.91 \mathrm{nM})$ of the small-molecule GLK inhibitor verteporfin also inhibits MAP4K1 (HPK1) [34], indicating that verteporfin is a GLK and HPK1 dual inhibitor. Because HPK1 is a negative regulator of T-cell receptor signaling $[29,54]$ and B-cell receptor signaling [55], HPK1 inhibitors may be used as immune-boosting agents for anti-tumor immunity or vaccination [1]. Thus, combination treatments of HPK1 inhibitors with antiPD-1 antibody may be effective for cancer combination immunotherapy. Notably, cancer immunotherapy usually induces autoimmune responses, which would be potentially reduced by a GLK inhibitor. Thus, verteporfin is also likely to be a potential therapeutics for cancer immunotherapy due to its suppressive effects on both cancer progression and autoimmune diseases. Furthermore, screening GLK inhibitors would help identification of GLK and HPK1 dual inhibitors for cancer immunotherapy in the future.

\section{GLK deficiency results in prevention of aging}

Besides cell growth, cell proliferation, and cell migration, GLK also regulates animal lifespan. GLK deficiency in Caenorhabditis elegans results in an expansion of the worm lifespan [56]. Similarly, GLK-deficient mice show a significant extension of lifespan [34]. The phenotypes of GLK-deficient mice are normal and healthy. The serum levels of proinflammatory cytokines are increased in aged wild-type mice, but are decreased in aged GLK-deficient mice [34]. Chronic inflammation plays a critical role in the aging process. Thus, expanded lifespan of GLKdeficient mice may be due to decreased inflammatory responses (inflamm-aging), suggesting that GLK inhibitor may have anti-inflamm-aging effect. Furthermore, these findings suggest that treatments of human patients using 
GLK inhibitors may have additional beneficial effects. Nevertheless, we could not rule out the possibility that GLK inhibitors may have potential side effects of attenuated immunity against microbial infections.

\section{Discussions and conclusions}

GLK overexpression in $\mathrm{T}$ cell is a critical pathogenic factor for development of autoimmune diseases. Deficiency of GLK or GLK-downstream molecules (such as PKC , AhR, $\mathrm{ROR}(\mathrm{t})$ inhibits disease severity in autoimmune disease mouse models, indicating that GLK signaling is a therapeutic target for autoimmune disease. GLK-induced AhR/ phospho-RORyt complex selectively stimulates IL-17A gene transcription; therefore, inhibition of GLK signaling or AhR/phospho-RORyt complex (such as verteporfin treatment) abolishes IL-17A production but maintains physiological functions of other cytokines. Moreover, $\mathrm{GLK}^{+}$Th17 cell can be used as a biomarker to help selection of the SLE patient subpopulation $\left(\mathrm{GLK}^{\text {high }} \mathrm{IL}-17 \mathrm{~A}^{\text {high }}\right.$ ) that is responsive to IL-17A blockade or GLK inhibitors, leading to precision medicine for SLE.

GLK induces cell growth of cancer cells [21, 24]; however, whole-body GLK transgenic mice do not spontaneously develop any cancer [26]. These findings suggest that GLK contributes to tumorigenesis after cancer initiation/transformation. Besides enhancement of cell growth, inhibition of cell autophagy by GLK signaling may be also involved in cancer progression [22]. GLK overexpression in cancer tissues is highly correlated with cancer recurrence $[24,35]$. In cancer cells, GLK directly phosphorylates and activates IQGAP1, resulting in induction of Cdc42-mediated cell migration and cancer metastasis [26]. Furthermore, cancer cell migration is blocked by inhibition of GLK activity or disruption of the GLKIQGAP1 complex [26]. Treatment of natural-product GLK inhibitors or overexpression of the miRNAs targeting GLK inhibits cancer cell migration and invasion in cancer cell lines [41, 42, 53]. The GLK-IQGAP1 complex formation and IQGAP1 Ser-480 phosphorylation in cancer cells are correlated with poor survival of human lung cancer patients. These findings suggest that GLK signaling or GLK-induced IQGAP1 phosphorylation is a prognostic biomarker and therapeutic target for cancer metastasis/ recurrence.

$\mathrm{T}$ cells from autoimmune disease patients display increased GLK mRNA levels, suggesting that transcription factors/repressors, histone-modifying enzymes, DNA methyltransferases, microRNAs, and/or long-non-coding RNAs (lncRNAs) may be responsible for GLK overexpression. The downregulation of three identified GLK miRNAs in cancer tissues $[37,41,42]$ supports that GLK overexpression in cancers is due to downregulation of the miRNAs that target GLK 3'UTR. In addition, sixteen 5' UTR SNPs, fifty 3'UTR SNPs, and eighty missense
SNPs of GLK in cancer patients can be found through NCBI (National Center for Biotechnology Information) website. Moreover, about 240 gene variants of GLK are detected in multiple cancers through COSMIC (Catalogue Of Somatic Mutations In Cancer) website (https:// cancer.sanger.ac.uk/cosmic/search?q=MAP4K3). One of these gene variants of GLK, GLK E351K [39], results in enhancement of GLK kinase activity in cancer cells [26]. Furthermore, GLK mRNA levels in cancer tissues of human NSCLC patients are comparable to those of normal adjacent tissues [35], suggesting that GLK protein stability is enhanced in lung cancer cells by an unknown regulatory mechanism. Collectively, it will be interesting to study whether other gene variants can result in induction of GLK mRNA levels, protein stability, or kinase activity in human autoimmune disease or cancer patients. Investigation of regulatory mechanisms of GLK overexpression in autoimmune disease $\mathrm{T}$ cells or cancer tissues may help identification of additional therapeutic targets for these diseases.

Collectively, overexpression of GLK induces autoimmune disease and cancer metastasis. Conversely, inhibition of GLK signaling attenuates disease progression of both autoimmune disease and cancer metastasis. Thus, GLK inhibitors could be useful therapeutics for autoimmune disease, as well as cancer recurrence without induction of autoimmune responses [57]. Furthermore, GLK deficiency results in extension of lifespan, suggesting that GLK inhibitors may also have anti-aging effects by attenuating inflammatory responses.

\section{Abbreviations}

4E-BP1: elF4E-binding protein 1; AOSD: Adult-onset Still's disease; APS: Astragalus polysaccharide; Cdc42: Cell division control protein 42; CIA: Collagen-induced arthritis; EAE: Experimental autoimmune encephalomyelitis; GCK: Germinal center kinase; GLK: GCK-like kinase; HCC: Hepatocellular carcinoma; HCPT: 10-hydroxycamptothecin; HGK: HPK1/ GCK-like kinase; HPK1: Hematopoietic progenitor kinase 1; IC50: Half maximal inhibitory concentration; IKK: IKB kinase; IQGAP1: IQ motif-containing GTPaseactivating protein 1; KHS: Kinase homologous to SPS1/Ste20; MAP4K: MAP kinase kinase kinase kinase; NSCLC: Non-small cell lung carcinoma; PKCO: Protein kinase C-theta; PTC: Papillary thyroid carcinoma; RA: Rheumatoid arthritis; S6K: S6 kinase; SLE: Systemic lupus erythematosus; SNP: Single nucleotide polymorphism; TFEB: Transcription factor EB

\section{Acknowledgments}

We thank Taiwan Bio-Development Foundation (TBF) for the award of the TBF Chair in Biotechnology to T.-H. T.

\section{Authors' contributions}

$\mathrm{H}-\mathrm{CC}$ and T-HT reviewed the literature cited and wrote the manuscript. Both authors read and approved the final manuscript.

\section{Funding}

This work was supported by grants from the National Health Research Institutes, Taiwan (IM-107-PP-01 and IM-107-SP-01, to T.-H. T.; IM-107-PP-06, to H.-C. C.) and Ministry of Science and Technology, Taiwan (MOST-106-2321B-400-013 to T.-H. T.).

Availability of data and materials

Data and materials related to this work are available upon request. 


\section{Ethics approval and consent to participate}

Not applicable.

\section{Consent for publication}

Not applicable.

\section{Competing interests}

The authors declare that they have no competing interests.

Received: 8 July 2019 Accepted: 1 October 2019

Published online: 22 October 2019

\section{References}

1. Chuang HC, Wang X, Tan TH. MAP4K family kinases in immunity and inflammation. Adv Immunol. 2016;129:277-314.

2. Chen Y-R, Tan T-H. Mammalian c-Jun N-terminal kinase pathway and STE20related kinases. Gene Ther Mol Biol. 1999;4:83-98.

3. Chen YR, Meyer CF, Tan TH. Persistent activation of c-Jun N-terminal kinase 1 (JNK1) in $\gamma$ radiation-induced apoptosis. J Biol Chem. 1996;271:631-4.

4. Chen YR, Wang X, Templeton D, Davis RJ, Tan TH. The role of C-Jun Nterminal kinase (JNK) in apoptosis induced by ultraviolet $\mathrm{C}$ and $\gamma$ radiation: duration of JNK activation may determine cell death and proliferation. J Biol Chem. 1996;271:31929-36.

5. Chen YR, Tan TH. The c-Jun N-terminal kinase pathway and apoptotic signaling. Int J Oncol. 2000;16:651-62.

6. MacCorkle RA, Tan TH. Mitogen-activated protein kinases in cell-cycle control. Cell Biochem Biophys. 2005;43:451-61

7. Hu MC, Qiu WR, Wang X, Meyer CF, Tan TH. Human HPK1, a novel human hematopoietic progenitor kinase that activates the JNK/SAPK kinase cascade. Genes Dev. 1996;10:2251-64.

8. Boomer JS, Tan TH. Functional interactions of HPK1 with adaptor proteins. J Cell Biochem. 2005:95:34-44.

9. Pombo CM, Kehrl JH, Sanchez I, Katz P, Avruch J, Zon LI, Woodgett JR, Force T, Kyriakis JM. Activation of the SAPK pathway by the human STE20 homologue germinal centre kinase. Nature. 1995;377:750-4.

10. Diener K, Wang XS, Chen C, Meyer CF, Keesler G, Zukowski M, Tan TH, Yao $Z$. Activation of the c-Jun N-terminal kinase pathway by a novel protein kinase related to human germinal center kinase. Proc Natl Acad Sci U S A. 1997;94:9687-92.

11. Yao Z, Zhou G, Wang XS, Brown A, Diener K, Gan H, Tan TH. A novel human STE20-related protein kinase, HGK, that specifically activates the c-Jun Nterminal kinase signaling pathway. J Biol Chem. 1999;274:2118-25.

12. Fiedler LR, Chapman K, Xie M, Maifoshie E, Jenkins M, Golforoush PA, Bellahcene M, Noseda M, Faust D, Jarvis A, et al. MAP4K4 inhibition promotes survival of human stem cell-derived cardiomyocytes and reduces infarct size in vivo. Cell Stem Cell. 2019;24:579-91 e512.

13. Tung RM, Blenis J. A novel human SPS1/STE20 homologue, KHS, activates Jun N-terminal kinase. Oncogene. 1997;14:653-9.

14. Dan I, Watanabe NM, Kobayashi T, Yamashita-Suzuki K, Fukagaya Y, Kajikawa E, Kimura WK, Nakashima TM, Matsumoto K, Ninomiya-Tsuji J, Kusumi A. Molecular cloning of MINK, a novel member of mammalian GCK family kinases, which is up-regulated during postnatal mouse cerebral development. FEBS Lett. 2000;469:19-23.

15. Fu CA, Shen M, Huang BC, Lasaga J, Payan DG, Luo Y. TNIK, a novel member of the germinal center kinase family that activates the C-Jun $\mathrm{N}$ terminal kinase pathway and regulates the cytoskeleton. J Biol Chem. 1999; 274:30729-37

16. Chen YR, Meyer CF, Ahmed B, Yao Z, Tan TH. Caspase-mediated cleavage and functional changes of hematopoietic progenitor kinase 1 (HPK1). Oncogene. 1999;18:7370-7.

17. Salvesen GS, Dixit VM. Caspases: intracellular signaling by proteolysis. Cell. 1997:91:443-6.

18. Yan L, Mieulet V, Burgess D, Findlay GM, Sully K, Procter J, Goris J, Janssens V, Morrice NA, Lamb RF. PP2A T61 epsilon is an inhibitor of MAP4K3 in nutrient signaling to mTOR. Mol Cell. 2010;37:633-42.

19. Resnik-Docampo M, de Celis JF. MAP4K3 is a component of the TORC1 signalling complex that modulates cell growth and viability in Drosophila melanogaster. PLoS One. 2011;6:e14528.

20. Muthaiyan Shanmugam M, Bhan P, Huang HY, Hsieh J, Hua TE, Wu GH, Punjabi H, Lee Aplicano VD, Chen CW, Wagner OI. Cilium length and
Intraflagellar transport regulation by kinases PKG-1 and GCK-2 in Caenorhabditis elegans sensory neurons. Mol Cell Biol. 2018;38:e00612-17.

21. Findlay GM, Yan L, Procter J, Mieulet V, Lamb RF. A MAP4 kinase related to Ste20 is a nutrient-sensitive regulator of mTOR signalling. Biochem J. 2007; 403:13-20.

22. Hsu CL, Lee EX, Gordon KL, Paz EA, Shen WC, Ohnishi K, Meisenhelder J, Hunter T, La Spada AR. MAP4K3 mediates amino acid-dependent regulation of autophagy via phosphorylation of TFEB. Nat Commun. 2018;9:942.

23. Chuang HC, Lan JL, Chen DY, Yang CY, Chen YM, Li JP, Huang CY, Liu PE, Wang $X$, Tan TH. The kinase GLK controls autoimmunity and NF-KB signaling by activating the kinase PKC- $\theta$ in T cells. Nat Immunol. 2011;12:1113-8.

24. Ho CH, Chuang HC, Wu IC, Tsai HW, Lin YJ, Sun HY, Young KC, Chiu YC, Cheng PN, Liu WC, et al. Prediction of early hepatocellular carcinoma recurrence using germinal center kinase-like kinase. Oncotarget. 2016;7: 49765-76.

25. Hammond DE, Hyde R, Kratchmarova I, Beynon RJ, Blagoev B, Clague MJ. Quantitative analysis of HGF and EGF-dependent phosphotyrosine signaling networks. J Proteome Res. 2010;9:2734-42.

26. Chuang HC, Chang CC, Teng CF, Hsueh CH, Chiu LL, Hsu PM, Lee MC, Hsu $C P$, Chen YR, Liu YC, et al. MAP4K3/GLK promotes lung cancer metastasis by phosphorylating and activating IQGAP1. Cancer Res. 2019;79:4978-93.

27. Ensenat D, Yao Z, Wang XS, Kori R, Zhou G, Lee SC, Tan TH. A novel src homology 3 domain-containing adaptor protein, HIP-55, that interacts with hematopoietic progenitor kinase 1. J Biol Chem. 1999;274:33945-50.

28. Han J, Shui JW, Zhang X, Zheng B, Han S, Tan TH. HIP-55 is important for Tcell proliferation, cytokine production, and immune responses. Mol Cell Biol. 2005;25:6869-78.

29. Shui JW, Boomer JS, Han J, Xu J, Dement GA, Zhou G, Tan TH. Hematopoietic progenitor kinase 1 negatively regulates $T$ cell receptor signaling and $T$ cellmediated immune responses. Nat Immunol. 2007:8:84-91.

30. Wang X, Chuang HC, Li JP, Tan TH. Regulation of PKC- $\theta$ function by phosphorylation in T cell receptor signaling. Front Immunol. 2012;3:197.

31. Chen YM, Chuang HC, Lin WC, Tsai CY, Wu CW, Gong NR, Hung WT, Lan TH, Lan JL, Tan TH, Chen DY. Germinal center kinase-like kinase overexpression in T cells as a novel biomarker in rheumatoid arthritis. Arthritis Rheum. 2013; 65:2573-82.

32. Chen DY, Chuang HC, Lan JL, Chen YM, Hung WT, Lai KL, Tan TH. Germinal center kinase-like kinase (GLK/MAP4K3) expression is increased in adultonset Still's disease and may act as an activity marker. BMC Med. 2012;10:84.

33. Chuang HC, Tsai CY, Hsueh CH, Tan TH. GLK-IKK $\beta$ signaling induces dimerization and translocation of AhR-RORyt complex in IL-17A induction and autoimmune disease. Sci Adv. 2018;4:eaat5401.

34. Chuang HC, Chen YM, Chen MH, Hung WT, Yang HY, Tseng YH, Tan TH. AhR-RORyt complex is a therapeutic target for MAP4K3/GLK $K^{\text {high }} \mid \mathrm{L}-17 \mathrm{~A}^{\text {high }}$ subpopulation of systemic lupus erythematosus. FASEB J. 2019:33:11469-80.

35. Hsu CP, Chuang HC, Lee MC, Tsou HH, Lee LW, Li JP, Tan TH. GLKMMAP4K3 overexpression associates with recurrence risk for non-small cell lung cancer. Oncotarget. 2016;7:41748-57.

36. Varghese RT, Liang Y, Guan T, Franck CT, Kelly DF, Sheng Z. Survival kinase genes present prognostic significance in glioblastoma. Oncotarget. 2016;7: 20140-51.

37. Liu F, Yin R, Chen X, Chen W, Qian Y, Zhao Y, Jiang Y, Ma D, Hu T, Yu T, et al. Over-expression of miR-206 decreases the Euthyrox-resistance by targeting MAP4K3 in papillary thyroid carcinoma. Biomed Pharmacother. 2019;114:108605.

38. Lam D, Dickens D, Reid EB, Loh SH, Moisoi N, Martins LM. MAP4K3 modulates cell death via the post-transcriptional regulation of $\mathrm{BH}$-only proteins. Proc Natl Acad Sci U S A. 2009;106:11978-83.

39. Jones S, Zhang X, Parsons DW, Lin JC, Leary RJ, Angenendt P, Mankoo P, Carter $\mathrm{H}$, Kamiyama $\mathrm{H}$, Jimeno $\mathrm{A}$, et al. Core signaling pathways in human pancreatic cancers revealed by global genomic analyses. Science. 2008;321:1801-6.

40. Wright JH, Wang X, Manning G, LaMere BJ, Le P, Zhu S, Khatry D, Flanagan PM, Buckley SD, Whyte DB, et al. The STE20 kinase HGK is broadly expressed in human tumor cells and can modulate cellular transformation, invasion, and adhesion. Mol Cell Biol. 2003;23:2068-82.

41. Zhao B, Han H, Chen J, Zhang Z, Li S, Fang F, Zheng Q, Ma Y, Zhang J, Wu N, Yang Y. MicroRNA let-7c inhibits migration and invasion of human non-small cell lung cancer by targeting ITGB3 and MAP4K3. Cancer Lett. 2014;342:43-51.

42. Liu L, Lu L, Zheng A, Xie J, Xue Q, Wang F, Wang X, Zhou H, Tong X, Li Y, et al. MiR-199a-5p and let-7c cooperatively inhibit migration and invasion 
by targeting MAP4K3 in hepatocellular carcinoma. Oncotarget. 2017;8: 13666-77.

43. Wang L, Ma R, Kang Z, Zhang Y, Ding H, Guo W, Gao Q, Xu M. Effect of IL17A on the migration and invasion of NPC cells and related mechanisms. PLoS One. 2014;9:e108060.

44. Fabre JAS, Giustinniani J, Garbar C, Merrouche Y, Antonicelli F, Bensussan A. The interleukin-17 family of cytokines in breast cancer. Int J Mol Sci. 2018;19:3880.

45. Benevides $L$, da Fonseca DM, Donate PB, Tiezzi DG, De Carvalho DD, de Andrade JM, Martins GA, Silva JS. IL17 promotes mammary tumor progression by changing the behavior of tumor cells and eliciting tumorigenic neutrophils recruitment. Cancer Res. 2015;75:3788-99.

46. Marcotte D, Rushe M, Arduini RM, Lukacs C, Atkins K, Sun X, Little K, Cullivan M, Paramasivam M, Patterson TA, et al. Germinal-center kinase-like kinase co-crystal structure reveals a swapped activation loop and C-terminal extension. Protein Sci. 2017:26:152-62.

47. Chuang HC, Sheu WH, Lin YT, Tsai CY, Yang CY, Cheng YJ, Huang PY, Li JP, Chiu $L L$, Wang $X$, et al. HGK/MAP4K4 deficiency induces TRAF2 stabilization and Th17 differentiation leading to insulin resistance. Nat Commun. 2014;5:4602.

48. Chuang HC, Tan TH. MAP4K4 and IL-6 $6^{+}$Th17 cells play important roles in non-obese type 2 diabetes. J Biomed Sci. 2017;24:4.

49. Chuang HC, Wang JS, Lee IT, Sheu WH, Tan TH. Epigenetic regulation of HGK MAP4K4 in T cells of type 2 diabetes patients. Oncotarget. 2016;7:10976-89.

50. May-Dracka TL, Arduini R, Bertolotti-Ciarlet A, Bhisetti G, Brickelmaier M, CahirMcFarland E, Enyedy I, Fontenot JD, Hesson T, Little $K$, et al. Investigating small molecules to inhibit germinal center kinase-like kinase (GLK/MAP4K3) upstream of PKC $\theta$ phosphorylation: potential therapy to modulate T cell dependent immunity. Bioorg Med Chem Lett. 2018;28:1964-71.

51. Newman DK. Photodynamic therapy: current role in the treatment of chorioretinal conditions. Eye. 2016;30:202-10

52. Huggett MT, Jermyn M, Gillams A, lling R, Mosse S, Novelli M, Kent E, Bown SG, Hasan T, Pogue BW, Pereira SP. Phase I/II study of verteporfin photodynamic therapy in locally advanced pancreatic cancer. $\mathrm{Br} J$ Cancer. 2014:110:1698-704.

53. Zhou Y, Hong T, Tong L, Liu W, Yang X, Luo J, Wang F, Li J, Yan L. Astragalus polysaccharide combined with 10-hydroxycamptothecin inhibits metastasis in non-small cell lung carcinoma cell lines via the MAP4K3/mTOR signaling pathway. Int J Mol Med. 2018;42:3093-104.

54. Wang X, Li JP, Chiu LL, Lan JL, Chen DY, Boomer J, Tan TH. Attenuation of T cell receptor signaling by serine phosphorylation-mediated lysine 30 ubiquitination of SLP-76 protein. J Biol Chem. 2012;287:34091-100

55. Wang X, Li JP, Kuo HK, Chiu LL, Dement GA, Lan JL, Chen DY, Yang CY, Hu $H$, Tan TH. Down-regulation of B cell receptor signaling by hematopoietic progenitor kinase 1 (HPK1)-mediated phosphorylation and ubiquitination of activated B cell linker protein (BLNK). J Biol Chem. 2012;287:11037-48.

56. Khan MH, Hart MJ, Rea SL. The role of MAP4K3 in lifespan regulation of Caenorhabditis elegans. Biochem Biophys Res Commun. 2012;425:413-8.

57. Tan TH, Chuang HC. MAP kinase kinase kinase kinase 3 (MAP4K3) as a biomarker and therapeutic target for autoimmune disease, cancer, inflammation and IL-17-associated disease. 2014. US Patent, 8,846,311 B2.

\section{Publisher's Note}

Springer Nature remains neutral with regard to jurisdictional claims in published maps and institutional affiliations.

Ready to submit your research? Choose BMC and benefit from:

- fast, convenient online submission

- thorough peer review by experienced researchers in your field

- rapid publication on acceptance

- support for research data, including large and complex data types

- gold Open Access which fosters wider collaboration and increased citations

- maximum visibility for your research: over $100 \mathrm{M}$ website views per year

At $\mathrm{BMC}$, research is always in progress.

Learn more biomedcentral.com/submissions 